

\title{
Étude de faisabilité d'une nouvelle technique de butée acétabulaire arthroscopique dans la prise en charge de l'instabilité de hanche modérément dysplasique
} Rémy Coulomb, Eric Wolff, Olivier Mares, Philippe Marchand, Pascal Kouyoumdjian

\section{To cite this version:}

Rémy Coulomb, Eric Wolff, Olivier Mares, Philippe Marchand, Pascal Kouyoumdjian. Étude de faisabilité d'une nouvelle technique de butée acétabulaire arthroscopique dans la prise en charge de l'instabilité de hanche modérément dysplasique. Revue de Chirurgie Orthopédique et Traumatologique, 2019, 105 (3), pp.299-303. 10.1016/j.rcot.2019.02.006 . hal-02135195

\author{
HAL Id: hal-02135195 \\ https://hal.science/hal-02135195
}

Submitted on 21 May 2019

HAL is a multi-disciplinary open access archive for the deposit and dissemination of scientific research documents, whether they are published or not. The documents may come from teaching and research institutions in France or abroad, or from public or private research centers.
L'archive ouverte pluridisciplinaire HAL, est destinée au dépôt et à la diffusion de documents scientifiques de niveau recherche, publiés ou non, émanant des établissements d'enseignement et de recherche français ou étrangers, des laboratoires publics ou privés. 


\title{
Étude de faisabilité d'une nouvelle technique de butée acétabulaire arthroscopique dans la prise en charge de l'instabilité de hanche modérément dysplasique ${ }^{\text {ir }}$
}

\author{
Feasibility of a new arthroscopic shelf acetabuloplasty technique for hip instability \\ related to moderate dysplasia
}

\author{
Rémy Coulomb , Eric Wolff*, Olivier Mares , Philippe Marchand, Pascal Kouyoumdjian \\ CHU de Carémeau, place du Pr Debré, 30029 Nimes cedex 9, France
}

\section{R É S U M É}

L'instabilité de hanche dysplasique est à l'origine de douleurs et de dérangements intra-articulaires. La pathogénie de cette symptomatologie est double : osseuse et capsulo-labrale. La technique que nous proposons permet une correction peu invasive de la dysplasie osseuse par la réalisation d'une butée acétabulaire, la réparation des lésions secondaires (labrale par suture, laxité capsulaire par capsulorraphie), et de la came fémorale éventuelle.

Mots clés :

Arthroscopie de hanche

Butée acétabulaire

Instabilité de hanche

Dysplasie modérée de hanche

\section{Introduction}

La prise en charge arthroscopique de la hanche dysplasique non arthrosique, entrant dans le concept de micro-instabilité, est un challenge. Son indication pour les dysplasies à angle $\mathrm{VCE}<15^{\circ}$ n'est plus recommandée [1-3]. Pour les dysplasies modérées ou « bordeline » (VCE compris entre $20^{\circ}$ et $25^{\circ}$ ), l'arthroscopie permettrait le traitement des lésions acquises avec de bons résultats à court terme [4]. Si la reconstruction labrale et capsulaire semble la règle, la prise en charge osseuse reste soumise à débat.

Les partisans de la butée osseuse rapportent de bons résultats à long terme dans les dysplasies centrées avec un angle VCE $>0$ et un stade arthrosique faible (Tönnis $\leq 2$ ). [5-9]. D'autres auteurs sont partisans d'une prise en charge plus invasive par ostéotomie périacétabulaire dont les complications peuvent s'élever à 10,8 \% [10].

Le but de ce travail était de décrire la faisabilité cadavérique d'une technique arthroscopique originale peu invasive, permettant

DOI de l'article original : https://doi.org/10.1016/j.otsr.2019.01.004.

is Ne pas utiliser, pour citation, la référence française de cet article, mais celle de l'article original paru dans Orthopaedics $\mathcal{E}$ Traumatology : Surgery E'Research, en utilisant le DOI ci-dessus.

* Auteur correspondant. Service de chirurgie orthopédique et traumatologique, CHU de Carémeau, place du Pr Debré, 30029 Nîmes, France.

Adresse e-mail : e.ericwolff@gmail.com (E. Wolff). de corriger les paramètres dysplasiques de la hanche par réalisation d'une butée acétabulaire, la réparation des lésions secondaires (labrale par suture, laxité capsulaire par capsulorraphie), et le traitement d'une came fémorale éventuelle.

\section{Technique chirurgicale}

L'étude chirurgicale a été menée au laboratoire d'anatomie de la faculté de Nîmes, sur 4 cadavres frais (8 hanches). L'installation était le décubitus dorsal et un système de traction permettait l'approche du compartiment central. Le matériel utilisé était une colonne d'arthroscopie haute-définition, un ancillaire spécifique d'arthroscopie de hanche Stryker ${ }^{\circledR}$ comprenant notamment une optique à $70^{\circ}$.

Une capsulotomie extra-articulaire, hanche en flexion de $20^{\circ}$, évitait l'usage d'un amplificateur de brillance [11]. Pour ce premier temps, les deux voies d'abord étaient : antéro-latérale (AL : voie optique ; $1 \mathrm{~cm}$ en avant et dessus du sommet du grand trochanter) et antérieure intermédiaire (MA, mid-anterior : instrumentale : $5 \mathrm{~cm}$ plus distale que la voie $\mathrm{AL}$, permettant la triangulation). Les voies antérieure et proximal mid-anterior (PMA) étaient utilisées comme voies accessoires durant la procédure (Fig. 1). L'exposition capsulaire était réalisée à la sonde de radiofréquence crochet Serfas XL Energy Stryker ${ }^{\circledR}$. La capsule était incisée dans l'axe du col. Le couteau motorisé Formula XL Blade Aggressive Plus Cutter Stryker ${ }^{\circledR}$ 





(EXO)

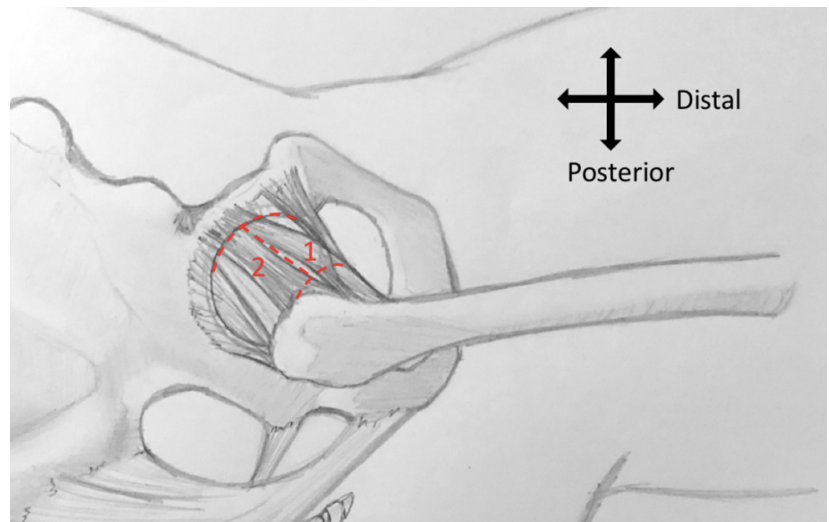

Fig. 2. Schéma représentant la capsulotomie en H. 1 : brin tractant le lambeau capsulaire médial par la voie antérieure ; 2 : brin tractant le lambeau capsulaire latéral par la voie antéro-latérale.

était utilisé à l'approche du labrum pour ne pas le léser. Des fils Premicron ${ }^{\circledR}$ permettaient d'écarter les deux lambeaux capsulaires (Fig. 2). Une capsulotomie en $\mathrm{H}$, avec caspulectomie médiale, assurait une visibilité complète de la jonction tête-col.

Une fémoroplastie, geste complémentaire éventuel, pouvait être ensuite réalisée à la fraise motorisée Formula XL Blade 8 Flute Round Burr Stryker ${ }^{\circledR}$, par voie antéro-latérale. La fémoroplastie était complétée en inversant les voies. Une traction permettait d'exposer le compartiment central. Après la résection du tendon réfléchi du droit antérieur une acétabuloplastie à la fraise créait la zone de réception de la butée (Fig. 3) ; une épaisseur de fraise dans ce contexte cadavérique non dysplasique.

Une incision centrée sur le tubercule du moyen fessier, permettait de prélever un greffon tri-cortical de $2 \times 2 \times 1 \mathrm{~cm}$. Celui-ci était méché de 4 trajets : 2 verticaux $(3,5 \mathrm{~mm})$ pour les vis d'ostéosynthèse et 2 horizontaux $(2 \mathrm{~mm})$ permettant le passage de fils, brins issus de la suture labrale, guidant sa descente sur le site receveur (Fig. 4 et 5). Dans une optique mini-invasive, la voie

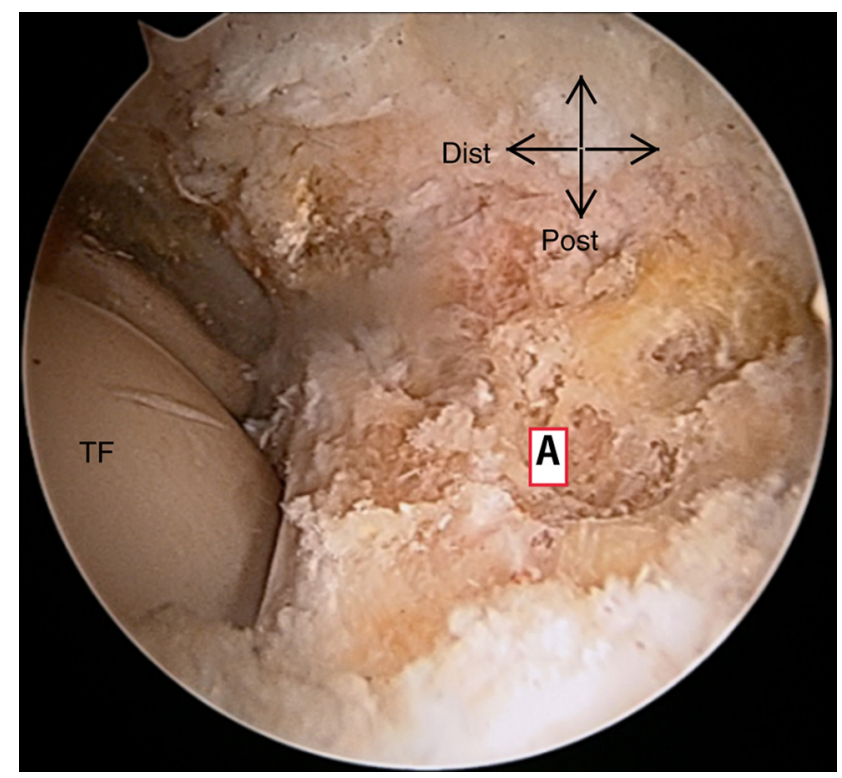

Fig. 3. Vue arthroscopique d'une acétabuloplastie. TF : tête fémorale ; A : acétabulum.

d'abord de prélèvement de la butée était ruginée en exopelvien, de manière sous-périostée, le long de la crête iliaque jusqu'au toit de l'acétabulum.

Le labrum déstabilisé par l'acétabuloplastie était fixé par points trans-osseux [12]. Le relâchement de la traction permettait d'observer l'effet joint du labrum réparé.

Les fils de suture labrale étaient passés au travers de la butée guidant sa descente par voie exopelvienne. Le positionnement de la butée, contrôlé sous arthroscopie était affleurant au labrum antérosupérieur. Deux broches guides étaient placées dans les tunnels préalables, permettant le vissage (AutofixStryker ${ }^{\circledR}$ diamètre 4) en compression de la butée (Fig. 6). Le vissage était effectué par voie 




Fig. 4. Photographie de la prise de greffe et voie exopelvienne. EAIS : épine iliaque antéro-supérieure.

antéro-latérale à $45^{\circ}$ par rapport à la ligne joignant les deux épines iliaques antéro-supérieures. Un temps de traction après passage des broches guides permettait de vérifier l'absence de matériel intra-articulaire.

Les fils tracteurs situés sur les berges médiales et latérales de la capsule étaient récupérés et noués entre eux selon une orientation verticale afin de permettre, avec la capsulectomie médiale, une retente du plan capsulaire médial (Fig. 7).

Après réalisation de la procédure, toutes les hanches ont été disséquées afin de valider le positionnement de la butée (Fig. 8). Le positionnement était affleurant au labrum sur les 8 hanches.

\section{Discussion}

Cette étude cadavérique montre la faisabilité d'une procédure arthroscopique peu invasive, assurant correction osseuse

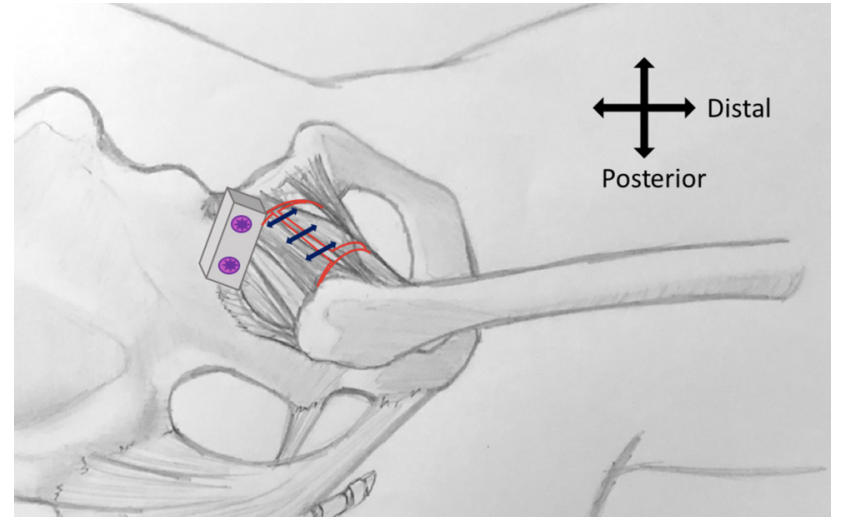

Fig. 7. Schéma représentant la retente capsulaire. En bleu : les points d'orientation verticale.

acétabulaire par butée, retente capsulo-ligamentaire et suture labrale dans les cas de dysplasie modérée entrant dans le concept global de micro-instabilité.

Ce travail présente plusieurs limites. Le défaut de souplesse tissulaire et l'absence de saignement sont des biais de l'étude cadavérique. La retente capsulaire était une limite technique, la butée gênant l'affrontement tissulaire et ainsi l'impossibilité de suture capsulaire médiale. Dans ce sens, afin de pallier à ce défaut de retente capsulaire, nous avons modifié notre capsulotomie afin de préserver la capsule médiale. Nous réalisons dorénavant une capsulotmie en $\mathrm{T}$ avec fermeture proximale sur ancres, en préservant au maximum l'insertion acétabulaire du lambeau médial, permettant ainsi une retente capsulaire plus efficace, ceci dans le but de diminuer le risque d'instabilité par défaut de fermeture médiale. De plus, ce travail n'a été effectué que sur huit hanches aux dépens de procédures longues. Enfin, dans un souci de limitation des coûts de l'étude, nous nous sommes passés d'amplificateur de brillance ainsi

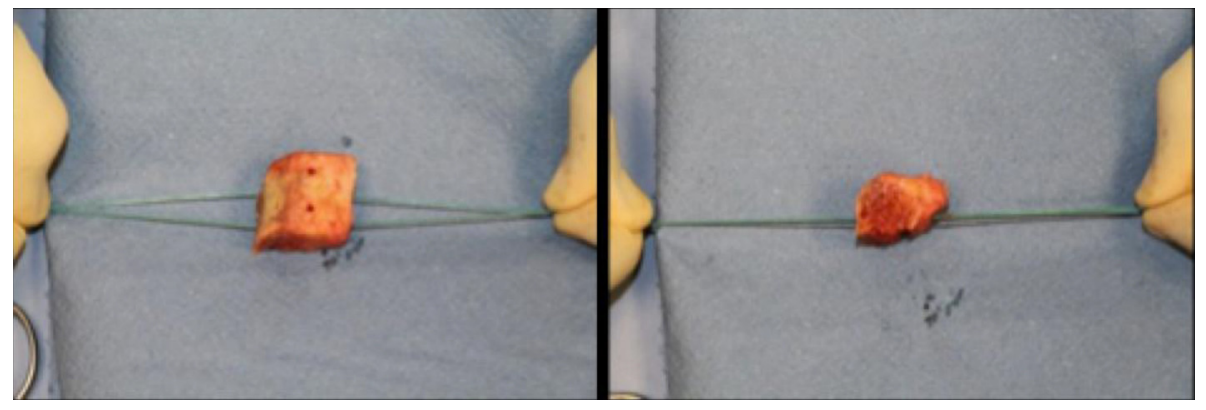

Fig. 5. Préparation de la butée osseuse.

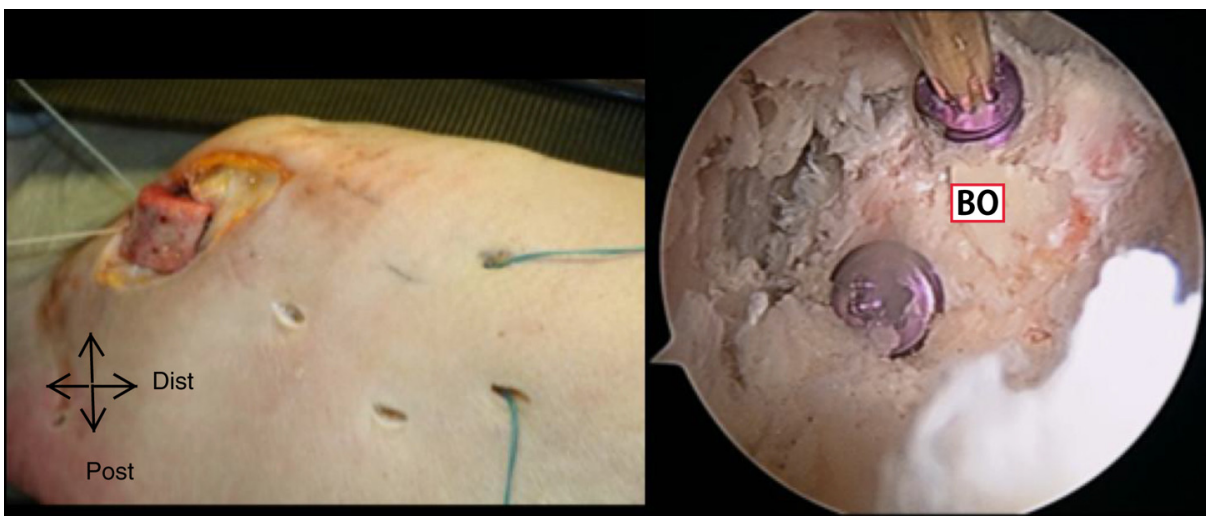

Fig. 6. Descente de la butée par voie exopelvienne le long des fils directeurs et vue arthroscopique du vissage de la butée osseuse. BO : butée osseuse. 


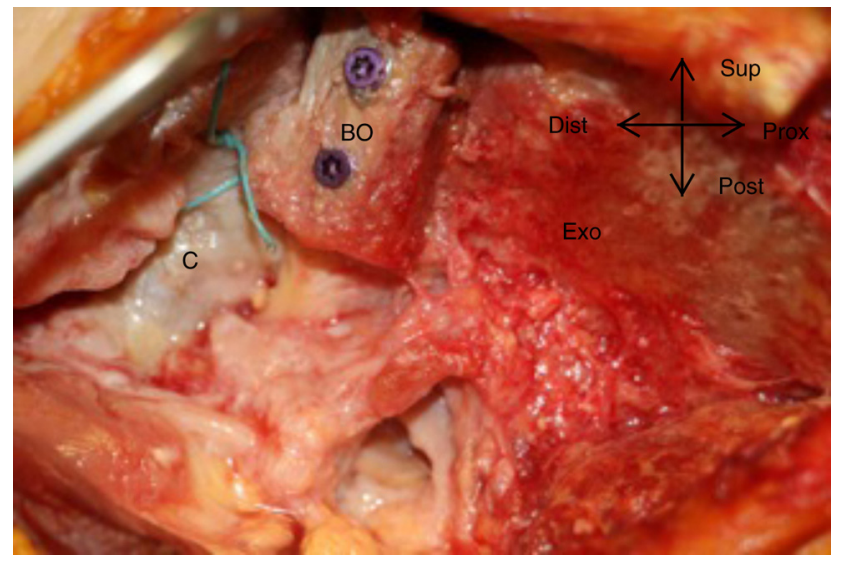

Fig. 8. Photographie d'une dissection complémentaire visualisant le positionnement de la butée. BO : butée osseuse ; $\mathrm{C}$ : capsule ; Exo : face exopelvienne de l'aile iliaque.

que d'ancres. Nous ne recommandons pas cette pratique, car elle entraîne un défaut de précision chirurgicale ainsi que des difficultés pratiques.

Selon Berton le taux de survie des butées de hanches à un recul de 16 ans minimum passerait de 83,3 \% à 15,2 \% en présence d'une lésion labrale. La préservation du labrum semble fondamentale et son état est corrélé avec le résultat fonctionnel de la butée [13]. Le choix d'une suture labrale trans-osseuse a été motivé par le coût des ancres difficiles à obtenir dans le cadre d'une étude cadavérique et représente une alternative de fixation[12,14,15]. Une étude récente réalisée par Uchida a objectivé la faisabilité de cette intervention entièrement sous endoscopie [16]. Toutefois les différences avec notre travail sont multiples. Une capsulotomie extra-articulaire permet de se passer d'un amplificateur de brillance, réduit la durée de traction et diminue les forces de traction nécessaires à la réalisation du temps central, sur cette chirurgie longue. Cependant, afin de s'assurer des corrections osseuses effectuées, le contrôle scopique reste d'utilisation plus que nécessaire. La voie d'abord exopelvienne, anatomiquement peu invasive, permet un placement simple et un contrôle visuel arthroscopique de son bon positionnement. Le décollement sous-périosté des glutéaux, limite leur traumatisme et ainsi le risque de formation d'ossifications hétérotopiques [17]. L'orientation supérieure de cette voie d'abord et la densité musculaire limitent la fuite de sérum intra-articulaire. La fixation par deux vis en compression semble plus stable qu'une impaction simple. Ainsi notre technique se situe comme une procédure intermédaire entre celle décrite par Uchida et la technique mini-invasive décrite par Chiron [18]. Dans notre pratique chirurgicale, nous réalisons actuellement nos butées de hanche avec des mini-Hueter sous contrôle scopique dont le but à terme est de rendre cette procédure entièrement endoscopique.

\section{Conclusion}

Notre technique peu invasive permet une prise en charge complète de l'instabilité de la hanche modérément dysplasique, mais nécessite une courbe d'apprentissage longue afin de diminuer la durée de certains temps opératoires complexes.

\section{Déclaration de liens d'intérêts}

Rémy Coulomb et Eric Wolff déclarent ne pas avoir de liens d'intérêts.

Olivier Mares déclare qu'il n'a pas de conflit d'intérêt pour cette étude, mais est consultant pour Newclip et Stryker.
Philippe Marchand et Pascal Kouyoumdjian déclarent ne pas avoir de conflit d'intérêt pour cette étude, mais sont consultants pour Stryker.

\section{Financement}

Aucun. Les auteurs déclarent avoir eut un prêt de matériel de la part de la société Stryker. Le laboratoire d'anatomie de l'université de Nîmes a mis à disposition les sujets cadavériques à titre gratuit.

\section{Contribution}

Rémy Coulomb : rôle substantiel dans la conception du projet et du protocole expérimental, l'acquisition des résultats ou l'analyse et l'interprétation des résultats, l'écriture et la révision critique.

Eric Wolff : rôle substantiel dans la conception du projet et du protocole expérimental, l'acquisition des résultats ou l'analyse et l'interprétation des résultats, l'écriture et la révision critique.

Olivier Mares a écrit la première version de l'article ou participé à la révision critique.

Philippe Marchand a écrit la première version de l'article ou participé à la révision critique.

Pascal Kouyoumdjian : rôle substantiel dans la conception du projet et du protocole expérimental, l'acquisition des résultats ou l'analyse et l'interprétation des résultats, l'écriture et la révision critique.

Tous les auteurs ont lu et valident la version finale et soumise du manuscrit.

\section{Appendix A. Matériel complémentaire}

Le matériel complémentaire accompagnant la version en ligne de cet article est disponible sur : https://doi.org/ 10.1016/j.rcot.2019.02.006.

\section{Références}

[1] Mei-Dan O, McConkey MO, Brick M. Catastrophic failure of hip arthroscopy due to iatrogenic instability: can partial division of the ligamentum teres and iliofemoral ligament cause subluxation? Arthroscopy 2012;28:440-5.

[2] Philippon MJ. The role of arthroscopic thermal capsulorrhaphy in the hip. Clin Sports Med 2001;20:817-30.

[3] Nawabi DH, et al. Outcomes after arthroscopic treatment of femoroacetabular impingement for patients with borderline hip dysplasia. Am J Sports Med 2016:44:1017-23.

[4] Domb BG, Stake CE, Lindner D, El-Bitar Y, Jackson TJ. Arthroscopic capsular plication and labral preservation in borderline hip dysplasia: two-year clinical outcomes of a surgical approach to a challenging problem. Am J Sports Med 2013;41:2591-8.

[5] Kerboull M. General therapeutic indications in congenital hip dislocation in adults. Acta Orthop Belg 1990;56:407-13.

[6] Migaud H, Chantelot C, Giraud F, Fontaine C, Duquennoy A, et al. Long-term survivorship of hip shelf arthroplasty and Chiari osteotomy in adults. Clin Orthop 2004;418:81-6

[7] Migaud H, Spiers A, Gougeon F, Pierchon F, Fontaine C, Duquennoy A. Outcome of hip shelf arthroplasty in adults after a minimum of 15 years of followup. Long term results and analysis of failures of 56 dysplastic hips. Rev Chir Orthopedique Reparatrice Appar Mot 1995;81:716-23.

[8] Rosset P, Heudel B, Laulan J, Garaud P, Favard L. Long-term evolution following shelf procedure for hip dysplasia in adults. Shelf survival analysis in 68 cases and retrospective review of 44 with at least 26 years follow-up. Acta Orthop Belg 1999;65:315-26.

[9] Hirose S, Otsuka H, Morishima T, Sato K. Long-term outcomes of shelf acetabuloplasty for developmental dysplasia of the hip in adults: a minimum 20-year follow-up study. J Orthop Sci 2011;16:698-703.

[10] Clohisy JC, et al. Patient-reported outcomes of periacetabular osteotomy from the prospective ANCHOR cohort study. J Bone Joint Surg Am 2017;99:33-41.

[11] Horisberger M, Brunner A, Herzog RF. Arthroscopic treatment of femoroacetabular impingement of the hip: a new technique to access the joint. Clin Orthop 2010;468:182-90.

[12] Pérez-Carro L, et al. Transosseous acetabular labral repair as an alternative to anchors. Arthrosc Tech 2015;4:e407-10 
[13] Berton C, Bocquet D, Krantz N, Cotten A, Migaud H, Girard J. Shelf arthroplasties long-term outcome: influence of labral tears. A prospective study at a minimal 16 years' follows up. Orthop Traumatol Surg Res 2010;96:753-9.

[14] Wheeler DJ, et al. Biomechanical comparison of transosseous versus suture anchor repair of the subscapularis tendon. Arthroscopy 2010;26:444-50.

[15] Lertwanich P, Ejnisman L, Philippon MJ. Comments on "Labral Base Refixation in the Hip: Rationale and Technique for an Anatomic Approach to Labral Repair". Arthroscopy 2011;27:303-4.
[16] Uchida S, et al. Endoscopic shelf acetabuloplasty combined with labral repair cam osteochondroplasty, and capsular plication for treating developmental hip dysplasia. Arthrosc Tech 2014;3:e185-91.

[17] Firoozabadi R, O’Mara TJ, Swenson A, Agel J, Beck JD, Routt M. Risk factors for the development of heterotopic ossification after acetabular fracture fixation. Clin Orthop Relat Res 2014;472:3383-8.

[18] Chiron P, Laffosse JM, Bonnevialle N. Shelf arthroplasty by minimal invasive surgery: technique and results of 76 cases. Hip Int 2007; 17:S72-82. 